J. Korean Math. Soc. 41 (2004), No. 5, pp. 921-931

\title{
THE EXPECTED INDEPENDENT DOMINATION NUMBER OF RANDOM DIRECTED ROOTED TREES
}

\author{
Junho Song and Changwoo Lee
}

\begin{abstract}
We derive a formula for the expected value $\mu(n)$ of the independent domination number of a random directed rooted tree with $n$ labeled vertices and determine the asymptotic behavior of $\mu(n)$ as $n$ goes to infinity.
\end{abstract}

\section{Introduction}

Let $D$ be a digraph. A subset $S$ of vertices of $D$ is a dominating set of $D$ if for each vertex $v$ not in $S$ there exists a vertex $u$ in $S$ such that $(u, v)$ is an arc of $D$. The domination number of $D$ is the number $\alpha(D)$ of vertices in any smallest dominating subset of vertices in $D$. A subset $I$ of vertices of $D$ is an independent set of $D$ if no two vertices of $I$ are joined by an arc in $D$. The independence number of $D$ is the number $\beta(D)$ of vertices in any largest independent subset of vertices in $D$. An independent dominating set of $D$ is an independent and dominating set of $D$. The independent domination number of $D$ is the number $\alpha^{\prime}(D)$ of vertices in any smallest independent dominating subset of vertices in $D$. For definitions not given here see, for example, [2] and [7].

There are $\left(\begin{array}{c}2 n \\ n\end{array}\right) /(n+1)$ binary trees $T$ with $2 n+1$ vertices. Let $\mu(2 n+1)$ denote the expected value of the independent domination number $\alpha^{\prime}(T)$ over the set of such binary trees. Lee showed in [5] that

$$
\mu(2 n+1)=\sum(k+1) 2^{k} \frac{\langle n\rangle_{k}}{\langle 2 n\rangle_{k}}
$$

Received August 20, 2003.

2000 Mathematics Subject Classification: Primary 05C69; Secondary 05 C05.

Key words and phrases: independence number, domination number, independent domination number, random directed rooted tree, expected value.

This research was partially supported by the research fund of the University of Seoul, 2002. 
for $n=0,1,2, \ldots$, where the inner sum is over all even integers $k$ with $1 \leq k \leq n$ and $\langle n\rangle_{k}$ denotes the falling factorial $\langle n\rangle_{k}=n(n-1) \cdots(n-$ $k+1)$, and that

$$
\frac{\mu(2 n+1)}{2 n+1} \longrightarrow \frac{1}{2}
$$

as $n \rightarrow \infty$. We want to do similar work for the expected independent domination number of directed rooted trees.

A directed rooted tree is an oriented rooted tree in which every direction is led away from the root. There are $n^{n-1}$ directed rooted trees $T$ with $n$ labeled vertices. Let $\mu(n)$ denote the expected value of the independent domination number $\alpha^{\prime}(T)$ over the set of such trees.

Our goal is to show that

$$
\mu(n)=\sum\left(\begin{array}{c}
n-1 \\
k
\end{array}\right) n^{-k}(k+1) !,
$$

where the sum is over all even integers $k$ with $0 \leq k \leq n-1$, and that

$$
\frac{\mu(n)}{n} \longrightarrow \frac{1}{2}
$$

as $n \rightarrow \infty$.

\section{Some lemmas}

An oriented tree is a tree in which each edge is assigned a unique direction. A digraph might have no independent dominating sets as we can see in 3-cycles. However, every oriented tree has a unique independent dominating set [5]. Therefore, we have following lemma.

LEMMA 1. Every directed rooted tree has a unique independent dominating set.

Let $y_{n}$ denote the number of directed rooted trees $T$ with $n$ labeled vertices. Clearly, $y_{1}=1$. If we remove the root $r$ of $T$, along with all arcs incident from $r$, we obtain a (possibly empty) ordered collection of disjoint directed rooted trees, or 1-branches, whose roots were originally joined from $r$. If we classify these trees according to the number of 1branches attached from the root, count the number of ways of selecting the root vertex and forming 1-branches on the remaining vertices, and bear in mind that the ordering of the 1-branches is immaterial, we find that

$$
y_{n}=n \sum_{j=1}^{n-1} \frac{1}{j !} \sum\left(\begin{array}{c}
n-1 \\
a_{1}, \ldots, a_{j}
\end{array}\right) y_{a_{1}} \cdots y_{a_{j}}
$$


for $n \geq 2$, where the inner sum is over all solutions in positive integers to the equation $a_{1}+\cdots+a_{j}=n-1$. If we let

$$
y=y(x)=\sum_{n=1}^{\infty} y_{n} \frac{x^{n}}{n !}
$$

be the exponential generating function for directed rooted trees, then it follows from equation (2.1) that

$$
y=x+x \sum_{n=1}^{\infty} \frac{y^{n}}{n !}=x e^{y} .
$$

This implies that

$$
y=\sum_{n=1}^{\infty} n^{n-1} \frac{x^{n}}{n !}
$$

by Lagrange's inversion formula. This, of course, is a well-known argument (see [1] or [p. 26, 7]).

On the other hand, we can find the exponential generating function $y$ for directed rooted trees using a slightly different approach. Let $T$ be a directed rooted tree with $n$ labeled vertices and root $r$, and let $T_{r}$ denote the directed rooted subtree of $T$ induced by $N^{+}[r]$, where $N^{+}[r]$ consists of the root $r$ and its outneighbors. Suppose the outdegree of $r$ is $k$. If we remove $T_{r}$ from $T$, along with all arcs incident with vertices in $T_{r}$, we obtain an ordered $k$-tuple of disjoint (possibly empty) collections of directed rooted trees, or 2-branches.

If we classify these trees according to the outdegree $k$ of the root $r$, then the generating function for ordered $k$-tuples of disjoint collections of 2-branches is $\left(e^{y}\right)^{k}$ and the generating function for $T_{r}$ is $(k+1)\left(x^{k+1} /(k+1) !\right)$. Note that different orderings of collections of 2 -branches yield different directed rooted trees. Thus the generating function for directed rooted trees is

$$
y=\sum_{k=0}^{\infty}\left(e^{y}\right)^{k} \cdot(k+1) \frac{x^{k+1}}{(k+1) !}=x e^{x e^{y}},
$$

which is equivalent to $y=x e^{y}$.

The following lemma is a straightforward consequence of the definition of 2-branch and Lemma 1.

LEMMA 2. Let $T$ be a directed rooted tree. Then the independent domination number of $T$ is one more than the sum of the independent domination numbers of all 2-branches of $T$. 
For $1 \leq k \leq n$, let $y_{n, k}$ denote the number of directed rooted trees with $n$ labeled vertices whose independent domination number is exactly $k$. Let

$$
Y=Y(x, z)=\sum_{n=1}^{\infty}\left(\sum_{k=1}^{n} y_{n, k} z^{k}\right) \frac{x^{n}}{n !} .
$$

It follows by a slight extension of the argument used to establish equation (2.4) that

$$
Y=z x e^{x e^{Y}} .
$$

The factor $z$ is present in equation (2.5) because of Lemma 2. Notice that $y=Y(x, 1)$.

Lemma 3. Let $\mu(n)$ denote the expected value of the independent domination numbers of the $y_{n}$ directed rooted trees with $n$ labeled vertices and define

$$
M(x)=\sum_{n=1}^{\infty} \mu(n) y_{n} \frac{x^{n}}{n !}=\sum_{n=1}^{\infty} \mu(n) n^{n-1} \frac{x^{n}}{n !} .
$$

Then we have

$$
\begin{aligned}
M(x) & =\frac{y}{1-y^{2}} \\
& =\sum_{k=0}^{\infty} y^{2 k+1} .
\end{aligned}
$$

Proof. It is easy to see that

$$
M(x)=\sum_{n=1}^{\infty} \mu(n) y_{n} \frac{x^{n}}{n !}=Y_{z}(x, 1) .
$$

If we differentiate both sides of equation (2.5) with respect to $z$, set $z=1$, use the fact that equations (2.2) and (2.4) are equivalent, and solve for $Y_{z}(x, 1)$, we find the required result.

\section{A formula for $\mu(n)$}

We know that $M(x)$ is the exponential generating function for the total sums of the independent domination numbers of directed rooted trees. Therefore, using power series expansion of $M(x)$ in $x$, we could find directly the expected value $\mu(n)$ of the independent domination 
numbers of directed rooted trees for small $n$. Actually, it follows from (2.8), (2.3), and routine use of Mathematica that

$$
\begin{aligned}
{[*] \cdots M(x)=} & x+2 \frac{x^{2}}{2 !}+15 \frac{x^{3}}{3 !}+136 \frac{x^{4}}{4 !}+1645 \frac{x^{5}}{5 !}+24336 \frac{x^{6}}{6 !} \\
& +426979 \frac{x^{7}}{7 !}+8658560 \frac{x^{8}}{8 !}+199234809 \frac{x^{9}}{9 !} \\
& +5128019200 \frac{x^{10}}{10 !}+145969492471 \frac{x^{11}}{11 !} \\
& +4552809182208 \frac{x^{12}}{12 !}+154404454932325 \frac{x^{13}}{13 !} \\
& +5656950010320896 \frac{x^{14}}{14 !}+222655633595044875 \frac{x^{15}}{15 !} \\
& +\cdots
\end{aligned}
$$

Here is a table for $\mu(n)$ and $\mu(n) / n$. The entries for $n \leq 5$ were verified by drawing all of the diagrams for directed rooted trees with up to 5 vertices.

\begin{tabular}{rrrrl}
\hline$n$ & \multicolumn{1}{c}{$y_{n}$} & \multicolumn{1}{c}{$\mu(n) y_{n}$} & $\mu(n)$ & $\mu(n) / n$ \\
\hline 1 & 1 & 1 & 1.00 & 1.0000 \\
2 & 2 & 2 & 1.00 & 0.5000 \\
3 & 9 & 15 & 1.66 & 0.5555 \\
4 & 64 & 136 & 2.12 & 0.5312 \\
5 & 625 & 1645 & 2.63 & 0.5264 \\
6 & 7776 & 24336 & 3.12 & 0.5216 \\
7 & 117649 & 426979 & 3.62 & 0.5184 \\
8 & 2097152 & 8658560 & 4.12 & 0.5160 \\
9 & 43046721 & 199234809 & 4.62 & 0.5142 \\
10 & 1000000000 & 5128019200 & 5.12 & 0.5128 \\
11 & see $[9]$ & see $[*]$ & 5.62 & 0.5114 \\
12 & see $[9]$ & see $[*]$ & 6.12 & 0.5100 \\
13 & see $[9]$ & see $[*]$ & 6.62 & 0.5083 \\
14 & see $[9]$ & see $[*]$ & 7.12 & 0.5061 \\
15 & see $[9]$ & see [*] & 7.62 & 0.5035 \\
\hline
\end{tabular}

Furthermore, we can derive a reasonably explicit formula for $\mu(n)$ as follows. 
THEOREM 4. The expected value of the independent domination numbers of directed rooted trees with $n$ labeled vertices is

$$
\mu(n)=\sum\left(\begin{array}{c}
n-1 \\
k
\end{array}\right) n^{-k}(k+1) !,
$$

where the sum is over all even integers $k$ with $0 \leq k \leq n-1$.

Proof. Let

$$
\begin{aligned}
& \phi(\lambda)=e^{\lambda} \quad \text { and } \\
& f(\lambda)=\frac{\lambda}{1-\lambda^{2}}=\sum_{i=0}^{\infty} \lambda^{2 i+1} .
\end{aligned}
$$

It follows from (2.2) and (3.1) that $y=x \phi(y)$ and from Lagrange's inversion formula (see [p. 17, 3]) that

$$
f(y)=f(0)+\sum_{n=1}^{\infty}\left[\left(\frac{d}{d \lambda}\right)^{n-1}\left(f^{\prime}(\lambda) \phi^{n}(\lambda)\right)\right]_{\lambda=0} \frac{x^{n}}{n !} .
$$

It follows from (2.7), (3.2), and (3.3) that

$$
\begin{aligned}
M(x) & =f(y) \\
& =\sum_{n=1}^{\infty}\left[\left(\frac{d}{d \lambda}\right)^{n-1}\left(f^{\prime}(\lambda) \phi^{n}(\lambda)\right)\right]_{\lambda=0} \frac{x^{n}}{n !} \\
& =\sum_{n=1}^{\infty}\left[\left(\frac{d}{d \lambda}\right)^{n-1}\left(\sum_{i=0}^{\infty}(2 i+1) \lambda^{2 i} \cdot e^{n \lambda}\right)\right]_{\lambda=0} \frac{x^{n}}{n !} \\
& =\sum_{n=1}^{\infty}\left[\sum_{k=0}^{n-1}\left(\begin{array}{c}
n-1 \\
k
\end{array}\right)\left(\sum_{i}(2 i+1\rangle_{k+1} \lambda^{2 i-k}\right) n^{n-1-k} e^{n \lambda}\right]_{\lambda=0} \frac{x^{n}}{n !} \\
& =\sum_{n=1}^{\infty} \sum\left(\begin{array}{c}
n-1 \\
k
\end{array}\right) n^{n-1-k}(k+1) ! \frac{x^{n}}{n !},
\end{aligned}
$$

where the inner sum is over all even integers $k$ with $0 \leq k \leq n-1$. Thus it follows from (2.6) and (2.3) that

$$
\begin{aligned}
M(x) & =\sum_{n=1}^{\infty} \mu(n) n^{n+1} \frac{x^{n}}{n !} \\
& =\sum_{n=1}^{\infty} \sum\left(\begin{array}{c}
n-1 \\
k
\end{array}\right) n^{n-1-k}(k+1) ! \frac{x^{n}}{n !},
\end{aligned}
$$


where the inner sum is over all even integers $k$ with $0 \leq k \leq n-1$. Comparing the coefficients of $x^{n} / n$ ! in (3.4) and (3.5), we obtain the required result.

\section{The asymptotic behavior of $\mu(n)$}

To determine the asymptotic behavior of $\mu(n) / n$, we need the following lemma [5].

LeMMA 5. Let $A(x)=\sum_{n=0}^{\infty} a_{n} x^{n}$ and $B(x)=\sum_{n=0}^{\infty} b_{n} x^{n}$ be power series with radii of convergence $\rho_{1} \geq \rho_{2}$, respectively. Suppose that $A(x)$ converges absolutely at $x=\rho_{1}$. Suppose that $b_{n}>0$ for all $n$ and that $b_{n-1} / b_{n}$ approaches a limit $b$ as $n \rightarrow \infty$. If $\sum_{n=0}^{\infty} c_{n} x^{n}=A(x) B(x)$, then $c_{n} \sim A(b) b_{n}$.

Now we can state the main result of this paper.

THEOREM 6. The expected value of the independent domination numbers of directed rooted trees with $n$ labeled vertices is

$$
\mu(n) \sim \frac{1}{2} n
$$

Proof. Let

$$
\begin{aligned}
& A(x)=\frac{y}{1+y}=\sum_{n=1}^{\infty} a_{n} x^{n} \text { and } \\
& B(x)=\frac{1}{1-y}=\sum_{n=0}^{\infty} b_{n} x^{n}
\end{aligned}
$$

where $y$ is the exponential generating function for directed rooted trees, that is, $y=x e^{y}=\sum_{n=1}^{\infty} n^{n-1} \frac{x^{n}}{n !}$. Using the same argument as in Lemma 4 , we obtain

$$
\begin{aligned}
a_{n} & =\frac{1}{n !} \sum_{k=0}^{n-1}(-1)^{k}\left(\begin{array}{c}
n-1 \\
k
\end{array}\right) n^{n-1-k}(k+1) ! \\
& =\frac{n^{n-1}}{n !} \sum_{k=0}^{n-1}(-1)^{k}\left(\begin{array}{c}
n-1 \\
k
\end{array}\right) n^{-k}(k+1) ! \\
b_{n} & =\frac{n^{n}}{n !}
\end{aligned}
$$


where we adopt the convention that $0^{0}=1$. It is easy to see that (2.3) is convergent for $|x| \leq e^{-1}$ and from (2.2) that $y\left(e^{-1}\right)=1$. Thus (4.1) and (4.2) are valid for $|x|<e^{-1}$ (see [pp. 112-114, 4]).

First, it follows from (4.5) that

$$
\frac{b_{n-1}}{b_{n}} \rightarrow \frac{1}{e}
$$

as $n \rightarrow \infty$.

Secondly, we want to show that (4.1) converges absolutely at $x=e^{-1}$ and that $A\left(e^{-1}\right)=1 / 2$. Let

$$
c_{k}=(-1)^{k}\left(\begin{array}{c}
n-1 \\
k
\end{array}\right) n^{-k}(k+1) !
$$

for $k=0, \ldots, n-1$ so that

$$
A\left(e^{-1}\right)=\sum_{n=1}^{\infty} a_{n} e^{-n}=\sum_{n=1}^{\infty} n^{n-1} \frac{e^{-n}}{n !} \sum_{k=0}^{n-1} c_{k} .
$$

Now we can show by an elementary but tedious argument that $\sum_{k=0}^{n-1} c_{k}$ is bounded; we omit the details of this argument. Moreover, we already know that

$$
\sum_{k=1}^{\infty} n^{n-1} \frac{e^{-n}}{n !}
$$

is absolutely convergent. Therefore, (4.6) is absolutely convergent (see [p. 73, 4]), that is, (4.1) converges absolutely at $x=e^{-1}$. Now, it follows from $y\left(e^{-1}\right)=1$ and (4.1) that $A\left(e^{-1}\right)=1 / 2$.

Finally, it follows from Lemma 5 that

$$
\mu(n) \frac{n^{n-1}}{n !} \sim A\left(e^{-1}\right) b_{n}=\frac{1}{2} \frac{n^{n}}{n !}
$$

since

$$
M=\sum_{n=1}^{\infty} \mu(n) n^{n-1} \frac{x^{n}}{n !}=\frac{y}{1-y^{2}}=A(x) B(x) .
$$

Hence, we obtain

$$
\mu(n) \sim \frac{1}{2} n
$$

as we required.

We know [6] that the expected independence number $\nu^{\prime}(n)$ of directed rooted trees with $n$ labeled vertices is

$$
\nu^{\prime}(n) \sim(.5671 \cdots) n .
$$


It is easy to see that $\alpha^{\prime}(T) \leq \beta(T)$ for any directed rooted tree $T$ with labeled vertices. Our result

$$
\mu(n) \sim .5 n
$$

is consistent with these two facts.

\section{A new proof for an equality}

The following equality appears in [8]:

$$
\sum_{k=0}^{n-1}\left(\begin{array}{c}
n-1 \\
k
\end{array}\right) n^{n-1-k}(k+1) !=n^{n},
$$

which was proved by using inverse pairs

$$
a_{n}=\sum_{k=0}^{n}\left(\begin{array}{l}
n \\
k
\end{array}\right) k^{n-k} b_{k}, \quad b_{n}=\sum_{k=0}^{n}(-1)^{k+n}\left(\begin{array}{l}
n \\
k
\end{array}\right) k n^{n-1-k} a_{k}
$$

and

$$
n !=\sum_{k=0}^{n}(-1)^{k+n}\left(\begin{array}{l}
n \\
k
\end{array}\right) k^{n} .
$$

In this section, we will give another proof for this equality with the aid of $M(x)$ in section 3 .

Since $y_{n, k}$ is actually the number of directed rooted trees with $n$ labeled vertices in which the number of vertices on even levels is $k$, $M(x)$ is the exponential generating function for the vertices on even levels of directed rooted trees with labeled vertices. Now, let $w_{n, k}$ be the number of directed rooted trees with $n$ labeled vertices in which the number of vertices on odd levels is $k$ and let

$$
W(x, z)=\sum_{n=1}^{\infty}\left(\sum_{k=1}^{n} w_{n, k} z^{k}\right) \frac{x^{n}}{n !} .
$$

Then, it follows that

$$
W(x, z)=x e^{Y}
$$

since the number of vertices on odd levels of a directed rooted tree $T$ is the sum of the vertices on even levels of all 1-branches of $T$. Let $\nu(n)$ denote the expected value of the number of vertices on odd levels of the $n^{n-1}$ directed rooted trees with $n$ labeled vertices and define

$$
N(x)=\sum_{n=1}^{\infty} \nu(n) n^{n-1} \frac{x^{n}}{n !} .
$$


Then, it follows from the same argument as in establishing the formula for $M(x)$ that

$$
\begin{aligned}
N(x) & =W_{z}(x, 1)=x e^{y} M(x)=y \frac{y}{1-y^{2}} \\
& =\sum_{n=1}^{\infty} \sum\left(\begin{array}{c}
n-1 \\
k
\end{array}\right) n^{n-1-k}(k+1) ! \frac{x^{n}}{n !},
\end{aligned}
$$

where the inner sum is over all odd integers $k$ with $0 \leq k \leq n-1$. Therefore, we obtain

$$
M(x)+N(x)=\sum_{n=1}^{\infty} \sum_{k=0}^{n-1}\left(\begin{array}{c}
n-1 \\
k
\end{array}\right) n^{n-1-k}(k+1) ! \frac{x^{n}}{n !} .
$$

On the other hand, $M(x)+N(x)$ is the exponential generating function for the total sums of the numbers of vertices in all directed rooted trees. Therefore, we obtain

$$
M(x)+N(x)=\sum_{n=1}^{\infty} n^{n} \frac{x^{n}}{n !} .
$$

Comparing the coefficients of $x^{n} / n !$ in (5.1) and (5.2), we obtain the equality.

We can observe that the expected value $\mu(n)$ of the number of vertices on even levels of the $n^{n-1}$ directed rooted trees with $n$ labeled vertices is

$$
\begin{aligned}
\mu(n) & =\sum\left(\begin{array}{c}
n-1 \\
k
\end{array}\right) n^{-k}(k+1) ! \\
& \sim \frac{1}{2} n,
\end{aligned}
$$

where the sum is over all even integers $k$ with $0 \leq k \leq n-1$ and that the expected value $\nu(n)$ of the number of vertices on odd levels of the $n^{n-1}$ directed rooted trees with $n$ labeled vertices is

$$
\begin{aligned}
\nu(n) & =\sum\left(\begin{array}{c}
n-1 \\
k
\end{array}\right) n^{-k}(k+1) ! \\
& \sim \frac{1}{2} n,
\end{aligned}
$$

where the sum is over all odd integers $k$ with $0 \leq k \leq n-1$. 


\section{References}

[1] A. Cayley, On the analytical forms called trees, Philos. Mag. 28 (1858), 374-378. [Collected Mathematical Papers, Cambridge 4 (1891), 112-115.]

[2] G. Chartrand and L. Lesniak, Graphs \& Digraphs, Wadsworth \& Brooks, Monterey, 1986.

[3] I. P. Goulden and D. M. Jackson, Combinatorial Enumeration, Wiley, New York, 1983.

[4] K. Knopp, Infinite Sequences and Series, Dover, New York, 1956.

[5] C. Lee, The expectation of independent domination number over random binary trees, Ars Combin. 56 (2000), 201-209.

[6] A. Meir and J. W. Moon, The expected node-independence number of random trees, Proc. Kon. Ned. v. Wetensch 76 (1973), 335-341.

[7] J. W. Moon, Counting Labelled Trees, Canadian Mathematical Congress, Montreal, 1970.

[8] J. Riordan, Combinatorial Identities, Robert E. Krieger, New York, 1979.

[9] N. J. A. Sloane and S. Plouffe, The Encyclopedia of Integer Sequences, Academic Press, San Diego, 1995.

Junho Song

Department of Mathematics

University of Seoul

Seoul 130-743, Korea

E-mail: jsong@uos.ac.kr

Changwoo Lee

Department of Mathematics

University of Seoul

Seoul 130-743, Korea

E-mail:chlee@uos.ac.kr 